\title{
GENERALIZED ROLEWICZ THEOREM FOR CONVEXITY OF HIGHER ORDER
}

\author{
ZOLTÁN Boros AND NOÉMI NAGY
}

Abstract. In this paper we prove that if a real function $f$ satisfies the inequality $\left[x_{0}, x_{1}, \ldots, x_{n+1} ; f\right]$ $+\varphi\left(x_{n+1}-x_{0}\right) \geqslant 0$ for all $x_{0}<x_{1}<\ldots<x_{n}<x_{n+1}$ with some fixed positive integer $n$ and nonnegative function $\varphi$ fulfilling $\lim _{h \rightarrow 0+} \varphi(h)=0$, then $f$ is convex of order $n$, i.e., $f$ satisfies the former inequality with $\varphi=0$ as well.

Mathematics subject classification (2010): 26A51, 39B62.

Keywords and phrases: Approximately convex functions, convexity of higher order.

\section{REFERENCES}

[1] R. P. BoAs And D. V. Widder, Functions with positive differences, Duke Math. J. 7 (1), (1940), 496-503.

[2] P. S. Bullen, A criterion for n-convexity, Pacific J. Math. 36 (1), (1971), 81-98.

[3] Z. CiESIELS KI, Some properties of convex functions of higher orders, Ann. Polon. Math. 7 (1), (1959), $1-7$.

[4] R. GER, Convex functions of higher order in Euclidean spaces, Ann. Polon. Math. 25 (3), (1972), 293-302.

[5] R. GER, n-convex functions in linear spaces, Aequationes Math. 10 (2-3), (1974), 172-176.

[6] R. GeR AND K. Nikodem, Strongly convex functions of higher order, Nonlinear Anal. 74 (2), (2011), 661-665.

[7] A. Gilányi And Zs. PÁles, On Dinghas-type derivatives and convex functions of higher order, Real Anal. Exchange 27 (2), (2001/2002), 485-494.

[8] A. Gilányi And Zs. PÁles, On convex functions of higher order, Math. Inequal. Appl. 11 (2), (2008), 271-282.

[9] E. HopF, Über die Zusammenhänge zwishen gewissen höheren Differenzenquotienten reeller Funktionen einer reellen Variablen und deren Differenzierbarkeitseigenschaften, Dissertation, FriedrichWilhelms-Universität Berlin, Berlin, (1926).

[10] M. KuCZMA, An Introduction to the Theory of Functional Equations and Inequalities, $2^{\text {nd }}$ Edition, Birkhäuser Verlag, Basel, (2009).

[11] N. E. NÖRLund, Vorlesungen über Differenzialrechnung, Berlin, (1924).

[12] A. Pinkus And D. Wulbert, Extending n-convex functions, Studia Math. 171 (2), (2005), 125152.

[13] T. Popoviciu, Sur quelques propriétés des fonctions d'une ou de deux variables réelles, Mathematica (Cluj) 8 (1934), (1), 1-85.

[14] T. Popoviciu, Les Fonctions Convexes, Hermann et Cie, Paris, (1945).

[15] A. W. Roberts And D. E. VArberg, Convex Functions, Academic Press, New York, London, (1973).

[16] S. Rolewicz, On $\gamma$-paraconvex multifunctions, Math. Japon. 24 (3), (1979), 293-300.

[17] S. Rolewicz, Paraconvex analysis, Control Cybernet. 34 (3), (2005), 951-965.

[18] JACEK TABOR AND JózeF TABOR, Paraconvex, but not strongly, Takagi functions, Control Cybernet. 41 (3), (2012), 545-559.

[19] Sz. WĄsowicz, Some properties of generalized higher-order convexity, Publ. Math. Debrecen 68 (1-2), (2006), 171-182. 
[20] Sz. WAssowicz, Support-type properties of convex functions of higher order and Hadamard-type inequalities, J. Math. Anal. Appl. 332 (2), (2007), 1229-1241. 